Regular article

\title{
AN INVERSE AGONIST OF THE HISTAMINE H3-RECEPTOR IMPROVES WAKEFULNESS IN NARCOLEPSY: STUDIES IN OREXIN-/- MICE AND PATIENTS
}

Jian-Sheng Lin ${ }^{1}$, Yves Dauvilliers ${ }^{2}$, Isabelle Arnulf ${ }^{3}$, Hélène Bastuji ${ }^{4}$, Christelle Anaclet ${ }^{1}$,

Régis Parmentier ${ }^{1}$, Laurence Kocher ${ }^{5}$, Masashi Yanagisawa ${ }^{6}$, Philippe Lehert ${ }^{7}$, Xavier

Ligneau $^{8}$, David Perrin ${ }^{8}$, Philippe Robert ${ }^{8}$, Michel Roux ${ }^{9}$, Jeanne-Marie Lecomte ${ }^{9}$ and JeanCharles Schwartz ${ }^{8,9}$

${ }^{1}$ INSERM-U628, 69373-Lyon, France; Université Claude Bernard, 69373-Lyon, France.

${ }^{2}$ Neurologie, INSERM-U888, CHU Hôpital Gui de Chauliac, 34925-Montpellier, France.

${ }^{3}$ Hôpital de la Pitié-Salpêtrière, 75651-Paris, France.

${ }^{4}$ Hôpital Neurologique, INSERM-U879, 69667-Bron, France.

${ }^{5}$ Centre Hospitalier Lyon Sud, 69495-Pierre-Bénite, France.

${ }^{6}$ Howard Hughes Medical Institute, University of Texas Southwestern Medical Center, Dallas, TX 75390-8584, U.S.A.

${ }^{7}$ Statistics Department, Faculty of Economics, FUCAM, Louvain Academy, Belgium.

${ }^{8}$ Bioprojet-Biotech, 35762-Saint Grégoire, France.

${ }^{9}$ Bioprojet, 75002-Paris, France.

Running title: H3-RECEPTOR INVERSE AGONIST \& NARCOLEPSY

Correspondence should be addressed to

Dr. Jian-Sheng Lin, D.Sc., M.D.

INSERM/UCBL-U628, Integrated Physiology of Brain Arousal Systems

Department of Experimental Medicine, Faculty of Medicine, Claude Bernard University, 8 avenue Rockefeller, 69373 Lyon Cedex 08, France.

Tel (33) 478777041

Fax (33) 478777150

Email lin@univ-lyon1.fr 


\section{Abbreviations}

DOPAC/DA, dihydroxyphenyl acetic acid/dopamine

DREM, direct REM sleep onset from wakefulness

ECG, electrocardiogram

EDS, excessive daytime sleepiness

EEG, electroencephalogram

EMG, electromyogram

ESS, Epworth sleepiness scale

HA, histamine

KO, knockout

MHPG/NA, 4-hydroxy-3-methoxy-phenylglycol/noradrenaline

MSLT, multiple sleep latency tests

MWT, maintenance of wakefulness test

REM, rapid eye movement

SWS, slow wave sleep

t-MeHA, tele-methylhistamine

WASO, wake-up episodes after sleep onset

WT, wild-type 


\section{Abstract}

Narcolepsy is characterized by excessive daytime sleepiness(EDS), cataplexy, direct onsets of rapid eye movement(REM) sleep from wakefulness(DREMs) and deficiency of orexins, neuropeptides that promote wakefulness largely via activation of histamine(HA) pathways. The hypothesis that the orexin defect can be circumvented by enhancing HA release was explored in narcoleptic mice and patients using tiprolisant, an inverse H3-receptor agonist. In narcoleptic orexin-/-mice, tiprolisant enhanced HA and noradrenaline neuronal activity, promoted wakefulness and decreased abnormal DREMs, all effects being amplified by co-administration of modafinil, a currently prescribed wake-promoting drug. In a pilot single-blind trial on 22 patients receiving a placebo followed by tiprolisant, both for one week, the Epworth Sleepiness Scale(ESS) score was reduced from a baseline value of 17.6 by 1.0 with the placebo $(p>0.05)$ and 5.9 with tiprolisant $(p<0.001)$. Excessive daytime sleep, unaffected under placebo, was nearly suppressed on the last days of tiprolisant-dosing. H3receptor inverse agonists could constitute a novel effective treatment of EDS, particularly when associated with modafinil.

Keywords: Narcolepsy; sleep disorders; histamine; orexin; H3-receptor inverse agonist; wakefulness; somnolence. 


\section{Introduction}

Narcolepsy is a rare disabling sleep disorder with a prevalence of $0.02-0.18 \%$; it is characterized by excessive daytime sleepiness (EDS) and abnormal rapid eye movement (REM or paradoxical) sleep manifestations, including cataplexy (sudden loss of muscle tone triggered by strong emotions), direct transitions from wakefulness to REM sleep (DREMs), sleep paralysis and hypnagogic hallucinations (Baumann et al., 2005; Mignot, 2005; Mignot and Nishino, 2005; Dauvilliers et al., 2007).

Recent data in animal models revealed that deficient orexin (also known as hypocretin) transmission causes narcolepsy (Lin et al., 1999; Chemelli et al., 1999). A marked decrease in orexin-A levels in the cerebrospinal fluid and in the number of orexin neurons in post-mortem brain tissues was also reported in patients with narcolepsy with cataplexy (Nishino et al., 2000). Orexins are excitatory peptides released by neurons from the lateral hypothalamus with widespread projections namely to aminergic neurons known to be involved in the control of wakefulness, e.g. histaminergic or noradrenergic neurons (Bayer et al., 2001; Eriksson et al., 2001; Bourgin et al., 2000). Since orexin neurons promote wakefulness, treatment by orexin administration would be desirable in narcoleptic patients but, as is often the case with peptides, it is not practically feasible for bioavailability reasons.

We reasoned that the lack of orexins could be circumvented by activating histaminergic neurons pharmacologically. Histaminergic neurons emanate from the tuberomammillary nucleus in the posterior hypothalamus and send excitatory terminals to the whole telencephalon. These neurons represent a major waking system in the brain (Schwartz et al., 1991; Lin, 2000) and appear necessary for the waking effect of orexins (Huang et al., 2001).

We examined this hypothesis, first using a reliable animal model of narcolepsy, the orexin-/-mouse, which displays DREMs, a characteristic symptom of the disease (Chemelli et al., 1999; Mignot, 2005; Fujiki, et al., 2006), then in narcoleptic patients, by testing the effects 
of tiprolisant (BF2.649), a potent and selective inverse agonist of the $\mathrm{H} 3$ receptors (Ligneau et al., 2007a and 2007b) in both proof-of-concept studies. This class of agents is known to promote wakefulness by inhibiting the constitutively active H3-autoreceptor, thereby enhancing histaminergic neuron activity and histamine (HA) release (Schwartz et al., 1991; Lin, 2000; Morisset et al., 2000; Vanni-Mercier et al., 2003; Parmentier et al., 2002, 2007).

\section{Material and Methods}

\section{Animals and Surgery}

All experiments followed EEC (86/609/EEC) and French National Committee (decree 87/848) directives and every effort was made to minimize the number of animals used and any pain and discomfort. Prepro-orexin knockout (KO) mice were offspring of the mouse strain generated by Chemelli et al. (1999) and kept on C57BL/6J genomic background. After backcrossing male orexin-/- mice and female wild-type (WT) mice for nine generations, the obtained orexin+/- mice were crossed to produce heterozygote and homozygote WT and KO littermates. To determine their genotypes with respect to orexin gene, tail biopsies were performed at the age of 4 weeks for DNA detection using PCR. The KO and WT alleles were amplified using a neo primer, 5'-CCGCTATCAGGACATAGCGTTGGC, and two genomic primers, 5'-GACGACGGCCTCAGACTTCTTGGG and 5'TCACCCCCTTGGGATAGCCCTTCC, the latter being common to KO and WT mice. The expected products were 600 and 400 base pairs for KO and WT, respectively.

At the age of 12 weeks and with a body weight of $30 \pm 2$ g, mice used for EEG and sleepwake studies were chronically implanted, under deep gas anesthesia using isoflurane $(2 \%, 200$ $\mathrm{ml} / \mathrm{min}$ ) and a TEM anesthesia system (Bordeaux, France), with six cortical electrodes (gold-plated tinned copper wire, $\varnothing=0.4 \mathrm{~mm}$, Filotex, Draveil, France) and three muscle electrodes (fluorocarbon-coated gold-plated stainless steel wire, $\varnothing=0.03 \mathrm{~mm}$, Cooner Wire 
Chatworth, CA, U.S.A.) to record the electroencephalogram (EEG) and electromyogram (EMG) and to monitor the sleep-wake cycle. All electrodes were previously soldered to a multi-channel electrical connector and each was separately insulated with a covering of heat-shrinkable polyolefin/polyester tubing. Cortical electrodes were inserted into the dura through 3 pairs of holes $(\varnothing=0.5 \mathrm{~mm})$ made in the skull, located respectively in the frontal (1 $\mathrm{mm}$ lateral and anterior to the bregma), parietal ( $1 \mathrm{~mm}$ lateral to the midline at the midpoint between the bregma and lambda), and occipital $(2 \mathrm{~mm}$ lateral to the midline and $1 \mathrm{~mm}$ anterior to the lambda) cortex. Muscle electrodes were inserted into the neck muscles. Finally, the electrode assembly was anchored and fixed to the skull with Super-Bond (Sun Medical Co., Shiga, Japan) and dental cement. This implantation allows stable and long-lasting polygraphic recordings (Parmentier et al., 2002).

\section{Polygraphic recording in the mouse and data acquisition and analysis}

After surgery, the animals were housed individually in transparent barrels $(\varnothing=20 \mathrm{~cm}$, height $=30 \mathrm{~cm}$ ) placed in an insulated sound-proof recording room maintained at an ambient temperature of $23 \pm 1{ }^{\circ} \mathrm{C}$ and on a $12 \mathrm{~h}$ light/dark cycle (lights-on at 7 a.m.), standard food and water being available ad libitum. A videocamera with infrared and digital time recording capabilities was set up in the recording room to observe and score, when necessary, the animals' behavior during the light or dark phase. After a 7-day recovery period, mice were habituated to the recording cable for 7 days before polygraphic recordings were started. Cortical EEG (contralateral frontoparietal leads) and EMG signals were amplified, digitized with a resolution of 256 and $128 \mathrm{~Hz}$, respectively, and computed on a CED 1401 Plus (Cambridge, UK). Using a Spike2 script and with the assistance of spectral analysis using the fast Fourier transform, polygraphic records were visually scored by 30 -sec epochs for wakefulness (W), slow wave sleep (SWS), and paradoxical or rapid eye movement (REM) 
sleep according to previously described criteria validated for mice (Valatx, 1971; Valatx and Bugat 1974, Parmentier et al., 2002). Direct REM sleep onset (DREMs) episodes, also called narcoleptic episodes or sleep onset REM periods by some authors (Chemilli et al., 1999; Mignot, 2005; Fujiki et al., 2006), were defined as the occurrence of REM sleep directly from $\mathrm{W}$, namely a REM episode that follows directly a wake episode lasting more than $60 \mathrm{sec}$ without being preceded by any cortical slow activity of more that $5 \mathrm{sec}$ during the $60 \mathrm{sec}$.

To assess changes in the qualitative aspects of $\mathrm{W}$ following different drug treatments, 200 consecutive cortical EEG samples of $30 \mathrm{sec}$ were extracted from the identified waking state 30 min after drug dosing. Analysis of EEG power spectral density was then performed within the $0.8-60 \mathrm{~Hz}$ frequency range using a fast Fourier transform routine. On the basis of visual and spectral analysis, samples containing artifacts occurring during active waking (with large movements) or immediately before or after other vigilance states (SWS, REMs or DREMs) were identified and omitted from the spectral analysis. Data were collapsed in 0.4 $\mathrm{Hz}$ bins. The power densities obtained during waking were summed over the $0.8-60 \mathrm{~Hz}$ frequency band (total power). To standardize the data, all power spectral densities at the different frequency ranges (e.g., $\delta 0.8-3 \mathrm{~Hz}, \theta 4-10 \mathrm{~Hz}, \alpha 10-15 \mathrm{~Hz}, \beta 20-30 \mathrm{~Hz}$ and $\gamma$ 30-60 $\mathrm{Hz}$ ) were expressed as a percentage relative to the total power of the same epochs (e.g. power in the fast rhythm ranges $(\beta+\gamma 20-60 \mathrm{~Hz}) /$ total power $(0.8-60 \mathrm{~Hz})=$ relative power in $20-60$ $\mathrm{Hz})$.

\section{Drug administration and experimental procedures in the mouse}

After recovery from the surgery and habituation to the recording cables, each mouse was subjected to a recording session of two continuous days, beginning at 7 a.m. During these periods, the animals were left undisturbed to obtain baseline parameters on the cortical EEG, sleep-wake cycle and circadian rhythm. After baseline recordings, animals were subjected to 
cortical EEG and sleep-wake recordings following administration of either a placebo (vehicle) or tiprolisant and/or modafinil. The vehicle consisted of $0.05 \mathrm{ml}$ of $\mathrm{NaCl}$ at $0.9 \%$ containing methylcellulose at $1 \%$. The doses used were $20 \mathrm{mg} / \mathrm{kg}$ for tiprolisant and $64 \mathrm{mg} / \mathrm{kg}$ for modafinil because of their equal potency in wake duration, as established during a pilot study. Drugs were dissolved in the vehicle, fresh before each administration, and were administered orally using a mouse gavage probe (20G, Phymep, Paris). All administrations were performed at 6.45 p.m. just before lights-off (7.00 p.m.), because orexin-/- mice display narcoleptic attacks only during lights-off phase (Chemilli et al., 1999 and our pilot study) and therefore this period is adequate for detecting any narcoleptic effect. The order of administration was randomized. Polygraphic recordings were made immediately after administration and were maintained during the whole lights-off period $(12 \mathrm{~h})$. Two administrations were separated by a period of 7 days (washout).

\section{Neurochemistry in the mouse}

WT and orexin-/- mice received drugs orally between 7.30 p.m. and 8.30 p.m. (dark period). They were decapitated 90 min after dosing. Blood was collected on heparin and stored at $+4^{\circ} \mathrm{C}$ before centrifugation $\left(12,000 \mathrm{rpm}, 20 \mathrm{~min},+4^{\circ} \mathrm{C}\right)$ to obtain plasma. Brain tissues were removed out, weighed and immediately frozen in liquid nitrogen. Plasma and tissues were stored at $-80^{\circ} \mathrm{C}$ until analysis. Whole brain tissues were homogenized in 10 volumes (w/v) of ice-cold $0.4 \mathrm{~N}$ perchloric acid with $2.7 \mathrm{mM}$ EDTA. The clear supernatant obtained after centrifugation $\left(2,000 \mathrm{x} \mathrm{g}, 30 \mathrm{~min},+4^{\circ} \mathrm{C}\right)$ was stored at $-80^{\circ} \mathrm{C}$ before measuring tele-methylhistamine (t-MeHA) by enzymoimmunoassay (Ligneau et al., 1998), monoamines and their metabolites by high performance liquid chromatography (HPLC) coupled to electrochemical detection (Ligneau et al., 2007) and drug levels by LC-MS/MS. 


\section{Tiprolisant and modafinil assays in the mouse}

Plasma and tissue supernatants samples were extracted using $1 \mathrm{ml}$ Oasis HLB SPE cartridges (Waters, Saint-Quentin-en-Yvelines, France) preconditioned with $0.5 \mathrm{ml}$ of methanol, followed by $0.5 \mathrm{ml}$ of water. Half a milliliter of the processed sample was pulled through the cartridge before washing with $0.5 \mathrm{ml}$ of $5 \%$ methanol in water and elution with $0.25 \mathrm{ml}$ of methanol. The methanolic eluant was dried at $43^{\circ} \mathrm{C}$ under vacuum, and the dried residue was reconstituted with $100 \mu 1$ of the LC mobile phase, and an aliquot of $20 \mu 1$ was injected into the LC-MS/MS system (Waters), a Quattro Micro system equipped with an Alliance 2795 pump and an electro-spray ionization (ESI) interface. The chromatographic separation was carried out on a X-Terra MS C18 reversed phase column $(1.5 \times 100 \mathrm{~mm}, 3.5$ $\mu \mathrm{m}$, Waters $)$ with a binary mobile phase $(0.02 \%$ trifluoroacetic acid in water $)$ in gradient conditions $(5-65 \%$ of acetonitrile in $6.5 \mathrm{~min})$. The flow $(0.6 \mathrm{ml} / \mathrm{min})$ was split $1: 3$ and introduced into the ESI source. Argon was used as collision gas at collision energies of $30 \mathrm{eV}$ for tiprolisant, $15 \mathrm{eV}$ for modafinil and $35 \mathrm{eV}$ for BF4.947 (i.e. (4[4-(2-fluorophenyl) piperazin-1-yl] butyl)-2-naphtalenecarboxamide) used as internal standard (IS). Ions of $\mathrm{m} / \mathrm{z}$ 296.1, 296 and 406.3 corresponding to the protonated molecules of tiprolisant, modafinil and IS, respectively, were selected as precursor ions. Peak area ratios of tiprolisant/IS or modafinil/IS over an effective calibration range of 1 to $100 \mathrm{ng} / \mathrm{ml}$ (with a limit of quantification of $1 \mathrm{ng} / \mathrm{ml}$ ) were used to determine tiprolisant and modafinil levels.

\section{Patients}

Twenty-two adult patients (14 men and 8 women) diagnosed with narcolepsy, comprising 21 with clear-cut cataplexy, were included in the study. Inclusion criteria for narcolepsy were the presence of EDS and of, at least, two direct onsets on REM sleep from wakefulness (DREMs) and a mean sleep latency of less than 8 min during the multiple sleep 
latency tests (MSLT) in accordance with the ICSD-2 criteria (The International Classification of Sleep Disorders, 2005). At the time of study, all patients had persistent EDS (recurrent sleep attacks almost daily for at least 3 months), in some cases despite their treatment with modafinil or methylphenidate (12 patients, i.e. 54.5\%). The remaining 10 patients had no treatment, although they suffered from moderate to severe somnolence and attacks of cataplexy. One of them also presented an obstructive sleep apnea syndrome that has been well controlled by continuous positive air pressure for several years. An ESS - a well-validated auto-questionnaire - with a score below 10 at baseline was an exclusion criterion. All patients were recruited from the sleep medicine practices of the 4 participating centers.

\section{Design of the clinical study}

Experiments were undertaken with the understanding and written consent of each patient; the protocol was approved by the Ethics Committee of Lyon (France) and was in compliance with national legislation and the Code of Ethical Principles for Medical Research Involving Human Subjects of the World Medical Association (Declaration of Helsinki).

This study was a pilot, prospective, comparative, sequential placebo-controlled, singleblind, multi-center study of a fixed dose of tiprolisant. All stimulant treatments were stopped at least three days before inclusion. In contrast, stable dose of anticataplectic medication for at least 3 months was tolerated (except tricyclic antidepressants, which were stopped before the study, since they display histamine $\mathrm{H} 1$ receptor antagonist activity and entail possible drug interaction with tiprolisant). Such medication (mainly venlafaxine) was taken by 8 patients, i.e. $36.3 \%$. Other medications such as central hypertensive drugs, codeine and dextropropoxyphen were forbidden. 
Following the completion of screening procedures, patients received once a day, for one week, capsules of placebo, followed, on the next week by capsules containing $40 \mathrm{mg}$ of tiprolisant taken in the morning, approximately 1 hour after awakening.

Study assessments were conducted during visits at baseline and at the end of weeks 1 and 2. Sleepiness assessed using the ESS (Johns, 1991) was the principal endpoint of the study. A sleep diary was used to record the number and duration of sleep episodes during the night and day and the global clinical impression of change was also completed. A plasma dosage of tiprolisant was performed 3 hours after the morning intake at the last visit. Adverse events were collected throughout the study. Investigators rated the severity and the relationship of each event to study medication or placebo. Safety measures included clinical laboratory tests (hematology and blood chemistry), vital signs, 12-lead ECG, and physical examinations conducted at study visits.

\section{Chemicals}

Drugs were expressed as equivalent of base. Their sources were as follows: tiprolisant [1-\{3-[3-(4-chlorophenyl)propoxy]propyl\}piperidine, hydrochloride] (Ligneau et al., 2007a and b) and BF30 [1-[3-(4-chlorophenyl)propyl]-4-phenylpiperazine, hydrochloride] were provided by Pr Schunack (Free University, Berlin, Germany) and Interquim (Barcelona, Spain), their synthesis will be described elsewhere. Modafinil [(diphenyl-methyl) sulphinyl-2acetamide] was either synthesized in the Bioprojet-Biotech laboratory or obtained from Cephalon (Modiadal ${ }^{\circledR}$, Paris, France). Dopamine, DOPAC, noradrenaline, MHPG (4hydroxy-3-methoxy-phenylglycol), t-MeHA, were from Sigma (Isle d'Abeau, France). All other chemicals were obtained from commercial sources and were of the highest purity available. 


\section{Statistical analysis}

In the animal study, statistics were performed using ANOVA followed by the appropriate post-hoc test as indicated in the legends of Figures 1 and 2. In the patient study, the principal endpoint was changes in the ESS score at the end of each week and secondary endpoints were number of diurnal somnolence episodes on the patient's diary, total nocturnal sleep time and global opinion of the investigators. Each patient acted as his/her own control. All analyses were performed on the intention-to-treat population, including all the 22 patients enrolled. One patient had to discontinue tiprolisant treatment after 3 days and was excluded from the efficacy analysis. The t-tests, which compared the variables measured during the tiprolisant period versus the placebo period, were performed on the paired data, only when both values were available.

\section{Results}

\section{Tiprolisant enhances histaminergic and noradrenergic neuron activity in orexin-/- mice}

In orexin-/- mice, HA neuron activity during the lights-off period (or dark phase during which rodents are active) was assessed by measuring the cortical level of tele-methyl histamine (t-MeHA), a major extracellular metabolite of HA. t-MeHA was slightly but not significantly decreased as compared to wild-type (WT) mice but nearly doubled upon treatment with tiprolisant at a supramaximal dose, as was the case in WT mice (Figure 1a). Modafinil, a drug currently used to decrease EDS in narcolepsy (Mignot and Nishino, 2005; Dauvilliers et al., 2007), enhanced t-MeHA levels to a lower extent (42\%) but, when associated to tiprolisant, the effect of the $\mathrm{H} 3$ receptor inverse agonist was strongly enhanced (216\% activation in orexin-/- mice). Noradrenergic neuron activity, assessed by the 4hydroxy-3-methoxy-phenylglycol/noradrenaline (MHPG/NA) ratio, was also slightly but not significantly decreased in orexin-/- mice and activated by tiprolisant in both WT and orexin-/- 
mice (43\% and 22\%, respectively). Modafinil alone did not modify noradrenergic activity, but enhanced the tiprolisant-elicited activation in both WT and orexin-/- mice (86 \% and $121 \%$, respectively; Figure 1b). Tiprolisant did not affect cortical dopaminergic activity, assessed by the dihydroxyphenyl acetic acid/dopamine (DOPAC/DA) ratio, or its decrease elicited by modafinil (Figure 1c); neither did it affect cortical serotoninergic activity, assessed by 5hydroxyindoleacetate/serotonine ratio (not shown). The apparently synergistic interactions between modafinil and tiprolisant did not result from changes in their plasma or brain pharmacokinetics since tiprolisant levels were unaffected and modafinil levels in plasma and brain were, actually, decreased by $18 \%$ and $49 \%$, respectively (Figure $1 \mathrm{~d}$ ).

\section{Tiprolisant enhances wakefulness and cortical arousal, decreases DREM episodes and synergizes with modafinil in orexin-/- mice.}

During the lights-off period, both tiprolisant and modafinil markedly enhanced wakefulness at the expense of slow wave sleep (SWS) and REM sleep (Figure 2A). However, unlike that of modafinil, the awakening effect of tiprolisant was characterized by an enhancement of cortical arousal, as revealed by the spectral analysis of the electroencephalogram (EEG) during waking (Figure 2B-C). Indeed, cortical fast $\theta$ activity (7$10 \mathrm{~Hz}$ ) and fast rhythms ( $\beta$ and $\gamma$ ranges, 20-60 Hz), both known to occur notably during exploration, attention or other cognitive activities, were enhanced markedly after tiprolisant, but not modafinil dosing (Figure 2C). In addition, whereas both tiprolisant and modafinil decreased total REM sleep, only tiprolisant considerably decreased both the number and duration of DREMs episodes (Figure 2D, E), mostly during the early hours after dosing.

Associating tiprolisant to modafinil resulted in a markedly enhanced and qualitatively modified action of the former on several aspects (Figure 2A-E). Firstly, there was an enhanced promotion of wakefulness, with a nearly total suppression of SWS and REM sleep 
during 3-6 $\mathrm{h}$ (Figure 2A, D). Moreover, there was not only an amplification of the enhancement of fast $\theta$ activity and fast rhythms observed with tiprolisant alone, but also a qualitative change manifested by a decrease in the slow component of the EEG (in the fast $\delta$ and slow $\theta$ ranges, namely $2-7 \mathrm{~Hz}$, see Figure 2B-C) during waking. Finally, the peak frequency of EEG power in $\theta$ activity was moved from $6.5 \mathrm{~Hz}$, seen with the vehicle or either drug administered alone, to $7.0 \mathrm{~Hz}$ seen with tiprolisant and modafinil association (Figure 2B). These effects, which did not occur with either drug administered alone, indicates a potent and enhanced cortical activation with suppression of cortical EEG signs of somnolence and drowsiness.

\section{Tiprolisant improves EDS in narcoleptic patients}

Demographic, clinical characteristics and current symptomatology are shown in Table 1. All patients presented persistent sleepiness at baseline (in some cases despite treatments), with an ESS above or equal to 10 (max 24). One patient complained of moderate headaches and rebound of cataplectic attacks after stopping clomipramine and methylphenidate treatments; this patient was under tiprolisant for 3 days when she discontinued the trial. Therefore, 21 patients completed the study.

We observed significant differences regarding the EDS with the medication as compared to placebo. Whereas the mean ESS score ( \pm S.D.) during the placebo period (16.55 $\pm 4.86)$ did not significantly differ from the ESS score at inclusion $(17.55 \pm 3.89)$, the score under tiprolisant treatment $(11.81 \pm 6.11)$ showed a $4.86 \pm 5.12$ point reduction $[95 \% \mathrm{CI}$ : $2.22,7.56]$ when compared to placebo $(\mathrm{p}=0.0006)$ and a $5.86 \pm 5.51$ point reduction $[95 \% \mathrm{CI}$ : $3.42,8.34]$ when compared to baseline $(p<0.0001)$ (Figure 3a). Besides, 9 patients were normalized (ESS $<11)$. The mean of total numbers of diurnal sleep episodes was significantly reduced between placebo $(14.32 \pm 9.54)$ and tiprolisant period $(9.99 \pm 9.45)$, a difference of 
4.36 [95\% CI: 3.35, 5.37] episodes per week ( $<<0.001$, Figure 3c). This effect became larger along with time of tiprolisant treatment $(2.0 \pm 2.1$ episodes recorded on day 1 vs. $0.3 \pm 1.0$ on day $7, \mathrm{p}=0.0046)$, whereas corresponding values did not change under placebo $(1.9 \pm 2.1$ and $1.7 \pm 2.2$ on days 1 and 7 , respectively), suggesting a delay in reaching the maximum effect of tiprolisant on this parameter (Figure 3b). The total nocturnal sleep duration was slightly reduced, although not significantly, between placebo and tiprolisant periods $(8.0 \pm 1.7 \mathrm{~h} / \mathrm{night}$ vs. $7.5 \pm 2.0$ ). The number and duration of wake-up episodes after sleep onset (WASO) did not significantly differ between the two periods, in spite of a tendency to increase (Figure 3df). The global opinion of the investigators on efficacy was significantly better after the tiprolisant period (moderate or good efficacy at $68.2 \%$ ) than after placebo (moderate or good efficacy at $9.1 \%$ only) $(p=0.0002)$.

Tiprolisant dosages were performed at the end of the treatment period with a median sampling time at $3.75 \mathrm{~h}$ after the last drug intake. The plasma level average was $100.6 \pm 78.1$ $\mathrm{ng} / \mathrm{ml}(\mathrm{n}=17)$. Elevated plasma levels $(>150 \mathrm{ng} / \mathrm{ml})$ were observed in 5 patients, in some cases related to the experience of adverse events occurring a few hours after drug intake.

No serious adverse event was noted during the study; 11 patients experienced 23 adverse events during tiprolisant treatment as compared to 7 patients experiencing 13 adverse events during placebo treatment. Among the 22 patients, the most frequent adverse events during the tiprolisant period were headache $(n=5)$, nausea $(n=4)$, insomnia $(n=2)$, malaise $(n=2)$ and sweating $(n=2)$. The majority $(95 \%)$ of adverse events occurred during the first 3 days of treatment. Seven adverse events rated severe occurred in 6 patients during tiprolisant period (and two under the placebo period), six of them likely or very likely related to the tiprolisant treatment, i.e. insomnia $(n=2)$, malaise sensation $(n=2)$, nausea $(n=1)$ and hallucination $(n=1)$. None of these adverse events led to treatment cessation and 21/22 patients fully complied with the prescribed treatments. However, one patient reduced the 
dosage of tiprolisant from $40 \mathrm{mg}$ /day to $10 \mathrm{mg}$ /day because of adverse events such as mild auditory hallucinations without hypnagogic or hypnopompic characteristics, insomnia and malaise, which then disappeared with the dose reduction and another patient stopped the treatment the day before the exit visit with the investigator. Finally, there were no differences among placebo and medication in clinical laboratory test results, vital signs, 12-lead electrocardiogram (ECG) or physical examinations.

\section{Discussion}

We have explored here for the first time, through preclinical and clinical studies, the potential utility of a novel class of psychotropic agents, the inverse agonists of the histamine (HA) H3-receptor, in the treatment of EDS in narcolepsy. This exploration was based upon the assumption that HA neurons are not defective and can still be activated by these agents in this pathology.

We found that, during the dark (active) period, the baseline activity of these neurons, as well as that of noradrenergic and serotoninergic neurons assessed by neurochemical markers (Figure 1 and Results) was not significantly reduced in the brain of orexin-/-mice as compared with those of WT mice. Similarly, H3-receptor mRNA levels in the hypothalamus, cortex and hippocampus were not modified in orexin-/-mice (our unpublished data). This was rather unexpected because orexin neurons project onto the nuclei of origin of these wake-promoting neurons, upon which the neuropeptides exert excitatory actions (Bayer et al., 2001; Brown et al.,2001; Eriksson et al.,2001; Bourgin et al.,2000; Sutcliffe and de Lecea, 2002). Nevertheless, MHPG/NA ratios seem normal in the autopsied brain of patients with narcolepsy (Kish et al.,1992). A decrease in the tissue HA content in the Doberman dog's brain was, however, reported (Nishino et al.,2001) but this animal model of narcolepsy differs 
from the present one, and changes in neurotransmitter storage pools cannot be readily interpreted in terms of changes in neuronal activity.

The H3-receptor inverse agonist was able to enhance HA and noradrenaline release to approximately the same extent in orexin-/-mice as in their WT counterparts, suggesting that the two ascending waking systems can be stimulated in this narcolepsy model. Activation of HA release by H3-receptor inverse agonists is well evidenced (Arrang et al.,1987; Morisset et al.,2000) and activation of noradrenergic neurons may result from excitation of locus coeruleus neurons by locally-released HA acting via postsynaptic H1- and H2-receptors (Bouthenet et al.,1988; Pillot et al.,2002; Korotkova et al.,2005) or, alternatively, blockade of presynaptic H3-heteroreceptors regulating noradrenaline release (Schlicker et al.,1992; Pillot et al.,2002).

Modafinil, a currently-prescribed wake-promoting drug with distinct mechanisms of action from those of $\mathrm{H} 3$ receptor inverse agonists (Parmentier et al.,2007), was used for comparison and co-administration with tripolisant. We found that modafinil enhanced tMeHA levels to a lesser extent than tiprolisant and that it did not significantly affect MHPG/NA ratio. Similarly, modafinil-induced waking was accompanied with c-fos labeling in the tuberomammillary nucleus but not the locus coeruleus in rats (Scammell et al.,2000) and its arousal-promoting action did not involve the presynaptic noradrenergic system in cats and mice (Lin et al.,1992; Wisor and Eriksson, 2005). Nevertheless, the change in t-MeHA was of modest amplitude and the wake-promoting effect of modafinil is unaffected in mice KO for the gene of the HA-synthesizing enzyme (Parmentier et al.,2007).

Interestingly, we evidenced a strong synergy between tiprolisant and modafinil regarding the activation of the histaminergic and noradrenergic systems; this was clearly independent of any pharmacokinetic drug interaction (see "Results"). The wake-promoting effect of modafinil does not depend on the HA-mediated transmission (Parmentier et 
al.,2007). Hence, it would activate histaminergic neurons in a way distinct from that of tiprolisant. Modafinil reduces the outflow of GABA in the posterior hypothalamus (Tanganelli et al., 1995; Ferraro et al.,1996), thereby diminishing a major inhibitory input from the anterior hypothalamus to the tuberomammillary neurons (Sherin et al.,1998), whereas tiprolisant reverses the constitutive activity of the H3-receptors, i.e. of a potent "brake" on HA release (Morisset et al.,2000). In turn, the synergy between the two drugs on noradrenergic neurons may simply reflect the enhanced HA release resulting from their association. It should be underlined that tiprolisant activates not only histaminergic and noradrenergic neurons but also other ascending wake-promoting systems projecting to cortical areas, i.e. cholinergic and dopaminergic neurons (Ligneau et al.,2007a and b).

Both tiprolisant and modafinil enhanced wakefulness during the lights-off (active) period in orexin-/-mice, as is observed in WT rodents (Scammell et al.,2000; Parmentier et al.,2007; Ligneau et al.,2007b) and cats (Lin et al.,1992; Ligneau et al.,1998,2007a). However, in orexin-/-mice, the action of tiprolisant clearly differed from that of modafinil. These mice display direct transitions from wakefulness to REM sleep (DREMs), the only characteristic narcoleptic phenotype identified so far in the mouse using objective EEG and EMG recordings. Chemelli et al. (1999) proposed that these episodes could correspond to cataplectic episodes in narcoleptic patients, this interpretation was, however, challenged (Siegel, 1999). Whereas both drugs attenuated almost equally SWS and, even more, REM sleep, only tiprolisant significantly decreased DREMs episodes. In agreement, modafinil does not affect cataplexy in narcoleptic patients (Billiard et al.,2006), whereas another H3-receptor antagonist was recently described to decrease cataplexy in Doberman dogs (Bonaventure et al.,2007) with no effect reported on wakefulness. Interestingly, we found here that combining tiprolisant to modafinil led to an almost total disappearance of SWS, REM sleep and DREMs episodes, an encouraging finding for their association in clinical practice. 
We propose that the unique effect of the H3-receptor inverse agonist on DREMs, as opposed to modafinil, as well as the striking synergy of their association, are related to their noradrenaline-releasing effect, not observed with modafinil alone. In agreement, noradrenergic neurons cease to discharge for an extended period during REM sleep, as well as during cataplectic episodes (Wu et al.,1999), and antidepressants with a preference towards the noradrenaline transporter display anti-cataplectic activity in patients (Baumann et al.,2005; Mignot and Nishino, 2005; Dauvilliers et al.,2007). Alternatively, the enhanced activation of histaminergic neurons with tiprolisant alone or, even more in association with modafinil, suppresses DREMs as histaminergic neurons also become silent during REM sleep (Vanni-Mercier et al.,2003; Takahashi et al.,2006) and abolition of HA-synthesis increases REM sleep (Parmentier et al.,2002).

One additional interesting observation with tiprolisant alone or, even more in association with modafinil, was the shift in the spectral distribution of cortical EEG power density toward high frequencies during waking (Figure $2 b, c)$. It may reflect the procognitive efficacy of these drugs on higher functions such as attention or learning (Ligneau et al,1998,2007a and b, Parmentier et al,2007), a property of potential therapeutic interest since narcoleptic patients often complain of deficits in attention and memory (Dauvilliers et al.,2007).

Starting from this promising preclinical data, a pilot trial with a simple design was undertaken to preliminarily assess the potential of tiprolisant in alleviating EDS in narcoleptic patients. This is the first clinical trial with any H3-receptor inverse agonist.

The trial consisted of a comparative, placebo-controlled, single-blind study in which patients received placebo on the first week and on the second week, a single dose of the drug (40 mg/day), a dosage based upon previous pharmacokinetic data, EEG recordings and attention test results in healthy volunteers (to be published). For safety reasons, patients were allowed to continue their anticataplectic treatments with antidepressants, which did not allow 
assessment of the effect of tiprolisant on cataplexy (that would have been difficult, anyway, in such a short trial). This simple design allowed rapid recruitment, as patients' participation was only two weeks, all of them being sure to receive the active compound (although they were not informed on the treatment sequence).

This phase II study, although performed on a small population ( $\mathrm{n}=22$ patients), showed a statistically-significant improvement of EDS with tiprolisant in comparison to placebo, of a magnitude suggesting its clinical significance. We may also emphasize that a major proportion of these patients (54.5\%) were severely affected with EDS, despite their previous treatments with modafinil or methylphenidate. All the measures of EDS studied were consistent with this improvement: ESS score, number and duration of diurnal sleep episodes and somnolence, and investigators' opinion on drug efficacy. The reduction of somnolence of 5.9 points on the ESS, as compared to baseline, appears equivalent to the results obtained after several months of treatment with modafinil (Baumann et al.,2005; Mignot and Nishino, 2005; Dauvilliers et al.,2007) and somewhat superior to the effect of sodium oxybate (Black and Houghton, 2006). Furthermore, somnolence and sleepiness episodes recorded in the patients' diaries progressively decreased and were almost suppressed at the end of the week, suggesting that tiprolisant required several days to achieve optimal efficacy, presumably in relationship with the 4-5 day delay to reach steady-state drug plasma levels as shown in healthy volunteers.

The drug was considered safe on the studied population. The overall frequency of adverse events was higher during the tiprolisant period (50\% of patients) in comparison to the placebo period (31.8\%). The most frequent adverse events were headache, nausea, insomnia, experienced mainly during the first three days of treatment. The most severe adverse events, i.e. insomnia and fainting sensation, probably related to an overdose of tiprolisant, given here 
in fixed dosage, could be substantially reduced, in future studies, by using an individual titration regimen and starting with a low dose of the drug.

For the sake of simplification, our pilot study did not measure sleepiness by more technical tools such as the maintenance of wakefulness test (MWT), often used to confirm the results of subjective measures performed in the patient's environment, i.e. ESS or sleep diaries. However MWT is correlated to ESS (Baumann et al.,2005; Mignot and Nishino, 2005; Dauvilliers et al.,2007) and this test, performed under artificial conditions, after a night hospitalisation, may not be representative of the patient's usual life conditions. The effect of the drug on cataplexy, hypnagogic hallucinations and sleep paralysis was not measured in this short study as the use of anticataplectic drugs was permitted.

In summary, both preclinical and clinical data indicate that an $\mathrm{H} 3$-receptor inverse agonist (tiprolisant) constitutes a novel effective treatment of EDS in narcolepsy. Tiprolisant at the dose of $40 \mathrm{mg}$ per day appears to be efficient in treating EDS of narcoleptic patents and to be well tolerated. These results need to be confirmed in larger populations and by using an optimized dosage and a double-blind design. The hypothesis that H3-receptor inverse agonists alone, or even more in association with modafinil, improve not only excessive daytime sleepiness but also other symptoms of narcolepsy such as cataplexy, remains to be scrutinized in appropriate clinical trials.

\section{Acknowledgments}

The authors wish to thank M.C. Dalin, G. Guidon, C. Buda, J.P. Sastre, M.D. Milcent, S. Dixon, S. Krief, C. Limoge for experimental or technical assistance. This work was supported by Bioprojet and Institut National de la Santé et de la Recherche Médicale (INSERM-U628). 


\section{References}

Arrang J.M., Garbarg M., Lancelot J.C., Lecomte J.M., Pollard H., Robba M., Schunack W., Schwartz J.C., 1987. Highly potent and selective ligands for histamine H3-receptors. Nature 327, 117-123.

Baumann C.R., Bassetti C.L., 2005. Hypocretins (orexins): clinical impact of the discovery of a neurotransmitter. Sleep Med Rev 9, 253-268.

Bayer L., Eggermann E., Serafin M., Saint-Mleux B., Machard D., Jones B., Muhlethaler M., 2001. Orexins (hypocretins) directly excite tuberomammillary neurons. Eur J Neurosci 14, 1571-1575.

Billiard M., Bassetti C., Dauvilliers Y., Dolenc-Groselj L., Lammers G.J., Mayer G., Pollmacher T., Reading P., Sonka K., 2006. EFNS guidelines on management of narcolepsy. Eur J Neurol 13, 1035-1048.

Black J., Houghton W.C., 2006. Sodium oxybate improves excessive daytime sleepiness in narcolepsy. Sleep 29, 939-946.

Bonaventure P., Letavic M., Dugovic C., Wilson S., Aluisio L., Pudiak C., Lord B., Mazur C., Kamme F., Nishino S. et al., 2007. Histamine $\mathrm{H}_{3}$ Receptor Antagonists: From target identification to drug leads. Biochem Pharmacol 73, 1084-1096.

Bourgin P., Huitron-Resendiz S., Spier A.D., Fabre V., Morte B., Criado J.R., Sutcliffe J.G., Henriksen S.J., de Lecea L., 2000. Hypocretin-1 modulates rapid eye movement sleep through activation of locus coeruleus neurons. J Neurosci 20, 7760-7765.

Bouthenet M.L., Ruat M., Sales N., Garbarg M., Schwartz J.C., 1988. A detailed mapping of histamine H1-receptors in guinea-pig central nervous system established by autoradiography with [125I]iodobolpyramine. Neurosci. 26, 553-600. 
Brown R.E., Sergeeva O.A., Eriksson K.S., Haas H.L., 2002. Convergent excitation of dorsal raphe serotonin neurons by multiple arousal systems (orexin/hypocretin, histamine and noradrenaline). J Neurosci 22, 8850-8859.

Chemelli R.M., Willie J.T., Sinton C.M., Elmquist J.K., Scammell T., Lee C., Richardson J.A., Williams S.C., Xiong Y., Kisanuki Y., Fitch T.E. et al., 1999. Narcolepsy in orexin knockout mice: molecular genetics of sleep regulation. Cell 98, 437-451.

Dauvilliers Y., Arnulf I., Mignot E., 2007. Narcolepsy with cataplexy. Lancet 369, 499-511.

Eriksson K.S., Sergeeva O., Brown R.E., Haas H.L., 2001. Orexin/hypocretin excites the histaminergic neurons of the tuberomammillary nucleus. J Neurosci 21, 9273-9279.

Ferraro L., Tanganelli S., O'Connor W.T., Antonelli T., Rambert F., Fuxe K., 1996. The vigilance promoting drug modafinil decreases GABA release in the medial preoptic area and in the posterior hypothalamus of the awake rat: possible involvement of the serotonergic 5-HT3 receptor, Neurosci Lett 220, 5-8.

Fujiki N., Cheng T., Yoshino F., Nishino S., 2006. Specificity of direct transition from wake to REM sleep in orexin/ataxin-3 narcoleptic mice, Sleep 29 (Abstract Suppl) A225.

Huang Z.L., Qu W.M., Li W.D., Mochizuki T., Eguchi N., Watanabe T., Urade Y., Hayaishi O., 2001. Arousal effect of orexin A depends on activation of the histaminergic system. Proc Natl Acad Sci USA 98, 9965-9970.

Johns M.W., 1991. A new method for measuring daytime sleepiness: the Epworth sleepiness scale. Sleep 14, 540-545.

Kish S.J., Mamelak M., Slimovitch C., Dixon L.M., Lewis A., Shannak K., DiStefano L., Chang L.J., Hornykiewicz O., 1992. Brain neurotransmitter changes in human narcolepsy. Neurology 42, 229-334.

Korotkova T.M., Sergeeva O.A., Ponomarenko A.A., Haas H.L., 2005. Histamine excites noradrenergic neurons in locus coeruleus in rats. Neuropharmacol 49, 129-134. 
Ligneau X., Landais L., Perrin D., Piriou J., Uguen M., Denis E., Robert Ph., Parmentier R., Anaclet C., Lin J.S. et al., 2007a. Brain histamine and schizophrenia: potential therapeutic applications of H3-receptor inverse agonists studied with BF2.649. Biochem Pharmacol 73, 1215-1224.

Ligneau X., Lin J.S., Vanni-Mercier G., Jouvet M., Muir J.L., Ganellin C.R., Stark H., Elz S., Schunack W., Schwartz J.C., 1998. Neurochemical and behavioral effects of ciproxifan, a potent histamine H3-receptor antagonist. J Pharmacol Exp Ther 287, 658-666.

Ligneau X., Perrin D., Landais L., Camelin J.C., Calmels T.P.G., Berrebi-Bertrand I., Levoin N., Capet M., Lecomte .J.M., Parmentier R. et al., 2007b. BF2.649, a non-imidazole inverse agonist/antagonist at human histamine $\mathrm{H}_{3}$ receptor: preclinical pharmacology. J. Pharmacol Exp Ther 320, 365-375.

Lin J.S., Roussel B., Akaoka H., Fort P., Debilly G., Jouvet M., 1992. Role of catecholamines in the modafinil and amphetamine induced wakefulness, a comparative pharmacological study in the cat. Brain Res 591, 319-326.

Lin J.S., 2000. Brain structures and mechanisms involved in the control of cortical activation and wakefulness, with emphasis on the posterior hypothalamus and histaminergic neurons. Sleep Med Rev 4, 471-503.

Lin L., Faraco J., Li R., Kadotani H., Rogers W., Lin X., Qiu X., Nishino S., Mignot E., 1999. The sleep disorder canine narcolepsy is caused by a mutation in the hypocretin (orexin) receptor 2 gene. Cell 98, 365-376.

Mignot E., 2005. Narcolepsy: pharmacology, pathophysiology and genetics. In: Kryger M.H., Roth T., Dement W.C. (Eds.), Principles and Practice of Sleep Medicine, $4^{\text {th }}$ edition, Elsevier Saunders, Philadelphia, pp. 761-779.

Mignot E., Nishino S., 2005. Emerging therapies in narcolepsy-cataplexy. Sleep 28, 754-763. 
Mochizuki T., Crocker A., McCormack S., Yanagisawa M., Sakurai T., Scammell T.E., 2004. Behavioral state instability in orexin knock-out mice. J Neurosci 24, 6291-6300.

Morisset S., Rouleau A., Ligneau X., Gbahou F., Tardivel-Lacombe J., Stark H., Schunack W., Ganellin C.R., Schwartz J.C., Arrang J.M., 2000. High constitutive activity of native $\mathrm{H} 3$ receptors regulates histamine neurons in brain. Nature 408, 860-864.

Nishino S., Fujiki N., Ripley B., Sakurai E., Kato M., Watanabe T., Mignot E., Yanai K., 2001. Decreased brain histamine content in hypocretin/orexin receptor-2 mutated narcoleptic dogs. Neurosci Lett 313, 125-128.

Nishino S., Ripley B., Overeem S., Lammers G.J., Mignot E., 2000. Hypocretin (orexin) deficiency in human narcolepsy. Lancet 355, 39-40.

Parmentier R., Anaclet C., Guhennec C., Brousseau E., Bricout D., Bozyczko-Coyne D., Spiegel K., Ohtsu H., Williams M., Lin J.S., 2007. The brain $\mathrm{H}_{3}$-receptor as novel therapeutic target for vigilance and sleep-wake disorders, Biochem Pharmacol 73, $1157-1171$.

Parmentier R., Ohtsu H., Djebbara-Hannas Z., Valatx J.L., Watanabe T., Lin J.S., 2002. Anatomical, physiological and pharmacological characteristics of histidine decarboxylase knock-out mice: evidence for the role of brain histamine in behavioural and sleep-wake control. J Neurosci 22, 7695-7711.

Pillot C., Heron A., Cochois V., Tardivel-Lacombe J., Ligneau X., Schwartz J.C., Arrang J.M., 2002. A detailed mapping of the histamine $\mathrm{H} 3$ receptor and its gene transcripts in rat brain. Neurosci 114, 173-193.

Scammell T.E., Estabrooke I.V., McCarthy M.T., Chemelli R.M., Yanagisawa M., Miller M.S., Saper C.B., 2000. Hypothalamic arousal regions are activated during modafinil-induced wakefulness. J Neurosci 20, 8620-8628. 
Schlicker E., Behling A., Lummen G., Gothert M., 1992. Histamine H3-receptor-mediated inhibition of noradrenaline release in the mouse brain cortex. Naunyn Schmiedebergs Arch Pharmacol 345, 489-493.

Schwartz J.C., Arrang J.M., Garbarg M., Pollard H., Ruat M., 1991. Histaminergic transmission in the mammalian brain. Physiol Rev 71, 1-51.

Sherin J.E., Elmquist J.K., Torrealba F., Saper C.B., 1998. Innervation of histaminergic tuberomammillary neurons by GABAergic and galaninergic neurons in the ventrolateral preoptic nucleus of the rat. J Neurosci 18, 4705-4721.

Siegel J.M., 1999. Narcolepsy: a key role for hypocretins (orexins). Cell 98, 409-412.

Sutcliffe J.G., de Lecea L., 2002. The hypocretins: setting the arousal threshold. Nature Rev Neurosci 3, 339-349.

Takahashi K., Lin J.S., Sakai K., 2006. Neuronal activity of histaminergic tuberomammillary neurons during wake-sleep states in the mouse. J Neurosci 26, 10292-10298.

Tanganelli S., Mora M.P., Ferraro L., Mendez-Franco J., Beani L., Rambert F., Fuxe K., 1995. Modafinil and cortical $\Upsilon$-aminobutyric outflow. Modulation by 5hydroxytryptamine neurotoxins. Eur J Pharmac 273, 63-71.

The International Classification of Sleep Disorders: Diagnostic and Coding Manual. $2^{\text {nd }}$ ed. Chicago, In: American Academy of Sleep Medicine, 2005.

Valatx J.L., Bugat R., 1974. Facteurs génétiques dans le déterminisme du cycle veillesommeil chez la souris. Brain Res 69, 315-330.

Valatx J.L., 1971. Enregistrement chronique des activités électriques cérébrales, musculaires et oculaires chez la souris. CR Séances Soc Bio. Fil 165, 112-115.

Vanni-Mercier G., Gigout S., Debilly G., Lin J.S., 2003. Waking selective neurons in the posterior hypothalamus and their response to histamine H3-receptor ligands: an electrophysiological study in freely moving cats. Behav Brain Res 144, 227-241. 
Wisor J.P., Eriksson K.S., 2005. Dopaminergic-adrenergic interactions in the wake promoting mechanism of modafinil. Neurosci 132, 1027-1034.

Wu M.F., Gulyani S.A., Yau E., Mignot E., Phan B., Siegel J.M., 1999. Locus coeruleus neurons: cessation of activity during cataplexy. Neurosci 91, 1389-1399.

\section{TABLE A, Characteristics of patients $(n=22)$ at inclusion}

Age (years, mean $\pm \mathrm{SD})$

BMI $\left(\mathrm{kg} / \mathrm{m}^{2}\right.$, mean $\left.\pm \mathrm{SD}\right)$

$\operatorname{Sex}(\mathrm{M} / \mathrm{F})$

Duration of narcolepsy (years)

Need for daily nap (\%)

Presence of cataplexy attacks at baseline (\%)

Presence of hypnagogic hallucinations at baseline (\%)

Presence of sleep paralysis at baseline (\%)

Presence of nocturnal sleep disruption at baseline (\%)

Epworth sleepiness scale score (mean \pm SD)
$41.6 \pm 13.2$

$28.1 \pm 6.3$

$14 / 8$

17.2

100

95.5

81.8

45.5

50.0

$17.5 \pm 3.9$ 


\section{Legends to figures}

\section{Figure 1}

Effects of tiprolisant and/or modafinil on indices of cortical aminergic neuron activity in WT and orexin-/- mice during the lights-off period. Tiprolisant $(20 \mathrm{mg} / \mathrm{kg})$ and/or modafinil $(64 \mathrm{mg} / \mathrm{kg})$ were administered orally to WT or orexin-/- mice between 7.30 p.m. and 8.30 p.m. (lights-off at 7.00 p.m.) and sacrificed 90 min later. Effects on t-MeHA level (a), MHPG/NA ratio (b) and DOPAC/DA ratio (c) in cerebral cortex. In (d), tiprolisant and modafinil concentrations in plasma and brain, determined by HPLC/MS, are depicted, indicating that the interactions observed between the effects of the two drugs are not attributable to pharmacokinetic factors. (A significant ANOVA followed by a post-hoc PLSD Fisher test indicates: $* \mathrm{p}<0.05, * * \mathrm{p}<0.01, * * * \mathrm{p}<0.001$ denoting significant differences versus corresponding saline-treated group; $£ \mathrm{p}<0.05$, $£ £ \mathrm{p}<0.01$ denoting significant differences between modafinil treated group and combination-treated group; \# $p<0.05$, \# \# $\mathrm{p}<0.01$ denoting significant differences between tiprolisant- and combination-treated groups. For catecholamines, $\mathrm{n}=6-13$ and 4-7 and for t-MeHA and drugs levels, $\mathrm{n}=14-21$ and 4-7 in WT and KO groups, respectively). 


\section{Figure 2}

Effects of tiprolisant and/or modafinil on sleep-wake cycles, cortical EEG and narcoleptic episodes in orexin-/-mice. Tiprolisant $(20 \mathrm{mg} / \mathrm{kg})$ and/or modafinil $(64 \mathrm{mg} / \mathrm{kg})$ were given just before lights-off at 7.00 p.m. Quantitative/qualitative analyses of subsequent sleep-wake parameters are shown: (A) representative examples of hypnograms showing the sleep-wake cycle of a $\mathrm{KO}$ mouse from $1 \mathrm{~h}$ before to $6 \mathrm{~h}$ after lights-off; (B) mean spectral distribution of cortical EEG power density during waking; data obtained by pooling 200 consecutive 30-sec wake epochs from each animal after drug administration using the fast Fourier transform routine within the $0.8-60 \mathrm{~Hz}$ frequency range. (C) compared drug effects on cortical EEG mean power ( \pm S.E.M.) at frequency ranges of 2-7 (including fast $\delta$ and slow $\theta$ components), 7-10 (fast $\theta$ activity) and 20-60 Hz (fast rhythms); (D) mean duration ( \pm S.E.M.) of sleep-wake stages and direct REM sleep onset periods (DREMs, defined as a direct transition from waking to REM sleep) during a 3 h recording and (E) mean cumulative number ( \pm S.E.M.) of DREMs episodes during a recording period of $0-6 \mathrm{~h}$ after compound administrations. Note that 1) DREMs episodes occurred during lights-off (A, D, E) in the orexin-/-mouse; 2) both tiprolisant and modafinil enhanced waking (W) and decreased both slow wave sleep (SWS) and rapid eye movement (REM) sleep (A, D); 3) tiprolisant, but not modafinil, enhanced cortical fast $\theta(7-10 \mathrm{~Hz})$ and fast rhythms $(20-60 \mathrm{~Hz})$, an effect amplified by co-administration with modafinil $(\mathbf{B}, \mathbf{C})$; 4) only co-administration of tiprolisant and modafinil decreased slow activities $(2-7 \mathrm{~Hz}, \mathbf{B}$ and $\mathbf{C})$ and increased the peak frequency of EEG power in $\theta$ activity, i.e., from 6.5 to $7 \mathrm{~Hz}(\mathbf{C})$; 5) Tiprolisant reduced DREMs episodes (duration and number) whereas modafinil allowed them to persist (A, D, E); and 6) coadministration of tiprolisant and modafinil resulted in a greater increase in waking and totally suppressed DREMs episodes (A, D, E). (* $\mathrm{p}<0.05, * * \mathrm{p}<0.01$; Dunnett's $\mathrm{t}$ test after significant ANOVA, $\mathrm{n}=12$ mice). 


\section{Figure 3}

Changes in Epworth sleepiness scale (ESS) scores, diurnal episodes of sleep and nocturnal sleep in narcoleptic patients receiving placebo and tiprolisant. Twenty-two patients orally received a placebo and $40 \mathrm{mg}$ tiprolisant successively and treatment-blinded, once a day, for one week. The sleepiness was scored according to the ESS scale at baseline and at the end of the placebo- and tiprolisant-treatment weeks (a). The patients filled in a diary in which they daily reported the number and duration of diurnal episodes of somnolence and sleep (b), nocturnal sleep duration and number and duration of wake-up episodes after sleep onset (WASO) (d). In (c) and (e), the weekly total of these values are reported. (n.s. p>0.05, * $\mathrm{p}=0.05, * * \mathrm{p}<0.0001)$. 